\title{
ON THE BIRTH AND DEATH OF ALGEBRAIC LIMIT CYCLES IN QUADRATIC DIFFERENTIAL SYSTEMS
}

\author{
JAUME LLIBRE, REGILENE OLIVEIRA, AND YULIN ZHAO
}

\begin{abstract}
In 1958 started the study of the families of algebraic limit cycles in the class of planar quadratic polynomial differential systems. In the present we known one familiy of algebraic limit cycles of degree 2 and four families of algebraic limit cycles of degree 4 , and that there are not limit cycles of degree 3 . These families of degree 2 and 4 are all the families of those degrees modulo an affine change of variables and a scaling of the time. We also know that there exist two families of algebraic limit cycles of degree 5 and one family of degree 6 , but we do not know if these families are all the families of degree 5 and 6 . Until today it is an open problem to know if there are algebraic limit cycles of degree higher than 6 inside the class of quadratic polynomial differential systems. Here we investigate the birth and death of all the known families of algebraic limit cycles of quadratic polynomial differential systems.
\end{abstract}

\section{Introduction AND STATEMENT OF THE MAIN RESUlts}

Consider the polynomial differential system in $\mathbb{R}^{2}$ given by

$$
\begin{aligned}
& \dot{x}=P(x, y), \\
& \dot{y}=Q(x, y),
\end{aligned}
$$

where the dot denotes derivative with respect to the time $t$. The number $m=\max \{\operatorname{deg} P$, $\operatorname{deg} Q\}$ is the degree of system (1).

When $m=2$ system (1) is a quadratic polynomial differential system or simply a quadratic system. Associated with system (1) we have the polynomial vector field

$$
\mathcal{X}=P(x, y) \frac{\partial}{\partial x}+Q(x, y) \frac{\partial}{\partial y} .
$$

An isolated periodic orbit in the set of all periodic orbits of system (1) is a limit cycle. Although there are hundreds of papers published on quadratic systems it remains many open questions on the existence, birth and death of the limit cycles of quadratic systems. In recent years a variety of methods were used to investigate the existence of limit cycles in quadratic systems.

2010 Mathematics Subject Classification. Primary: 34C07, 34C05, 37G15, 70K05.

Key words and phrases. Algebraic limit cycles; Hopf bifurcation; homoclinic orbits, heteroclinic orbits, quadratic polynomial differential systems. 
Let $\mathbb{R}[x, y]$ be the ring of the real polynomials in the variables $x$ and $y$. Given $f \in \mathbb{R}[x, y]$ the algebraic curve $f(x, y)=0$ is an invariant algebraic curve of system (1) if there exists $K \in \mathbb{R}[x, y]$ such that

$$
P \frac{\partial f}{\partial x}+Q \frac{\partial f}{\partial y}=K f
$$

The word invariant for the algebraic curve $f(x, y)=0$ comes from the fact on the points of this curve the condition (1) implies that the gradient of the curve is orthogonal to the vector field $\mathcal{X}$, in consequentely the algebraic curve is formed by orbits of the system. An invariant algebraic curve $f=0$ is called irreducible if the polynomial $f$ is irreducible

A limit cycle $\gamma$ of system (1) is called an algebraic limit cycle if it is contained in some irreducible algebraic invariant curve $f=0$. The degree of the algebraic limit cycle $\gamma$ is the degree of $f$.

Until now we only know few different families of planar quadratic systems having algebraic limit cycles. More precisely, eight families of quadratic systems having algebraic limit cycles of degree $n \leq 6$ are known. For degree 2 there is the family of quadratic systems known as Qin Yuan-Xun's system, see [23] (1958). It is known that there are no algebraic limit cycles of degree 3, see Evdokimenco [9, 10, 11] (1970-1979), or see Theorem 11 of [5] for a short proof. For degree 4 there are four families of quadratic systems having algebraic limit cycles, Yablonskii [24] (1966), Filiptsov [12](1973) and other two families described in [3] (2001), see also [4] (2003). These five families mentioned before provides all the algebraic limit cycles of degree two and four (up to affine change of variables and rescaling of the time). For degrees 5 and 6 we only know three one-parameter families of quadratic systems having algebraic limit cycles, they are described in [6] (2005) and in [1] (2019). It is unknown if these families are all the families of algebraic limit cycles of degree 5 and 6 modulo changes of variables. The question of whether quadratic systems can have algebraic limit cycles of degree higher than 6 remains open. See details about this subject in [19].

It is known that when a quadratic system has an algebraic limit cycle of degree 2 or 4 this is the unique limit cycle of the system, see [3]. Since the algebraic limit cycles of degree 5 and 6 have been obtained doing birational transformation of the quadratic systems having algebraic limit cycles of degree 4 (see [1] and [6]), also the known quadratic systems having algebraic limit cycles of degree 5 and 6 have a unique limit cycle. This fact simplifies the study of their phase portraits.

We note that the study of limit cycles is one of the more difficult problems of the qualitative theory of differential equation in the plane. See for example the 16-th Hilbert problem on the limit cycles of polynomial differential systems $[14,15]$, or for instance for limit cycles in continuous and discontinuous piecewise differential systems [13, 17].

An isolated equilibrium point $p$ of a quadratic system is a center if there is a neighborhood of $p$ filled up of periodic solutions, with the exception of $p$. 
The notion of Hopf bifurcation refers to the local birth or death of a periodic solution from an equilibrium point moving a parameter of the system. In a differential system a Hopf bifurcation typically occurs when a complex conjugate pair of eigenvalues of the linearised flow at a equilibrium point becomes purely imaginary. For more information about Hopf bifurcation see for instance the book of Kuznetsov [16]

An orbit $\gamma$ of system (1) is called a homoclinic orbit if there exists an equilibrium point $p$ of the system such that $p$ is the $\alpha$-limit and the $\omega$-limit of $\gamma$. Each homoclinic orbit together with its corresponding equilibrium point defines a homoclinic loop. A heteroclinic orbit is an orbit whose $\alpha$-limit and $\omega$-limit are two distinct equilibria. Several heteroclinic orbits connected forming a close curve define a heteroclinic loop.

The phase portraits of the quadratic systems are presented in the Poincaré disc, see section 2 for an introduction to the Poincaré disc and its local charts $U_{k}$ and $V_{k}$ for $k=1,2$.

In this article we investigate where born or death the algebraic limit cycles in the known families of planar quadratic systems. The main results of this article are the following four theorems.

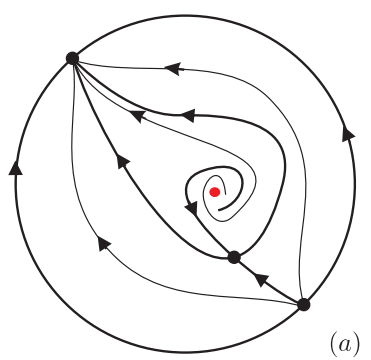

$b>-1 \quad r=0$

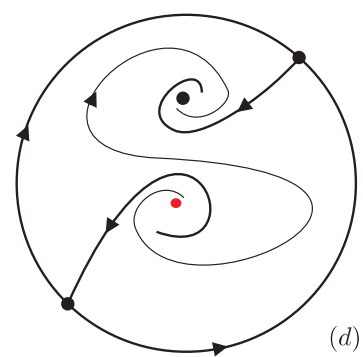

$b<-1 \quad r=0$

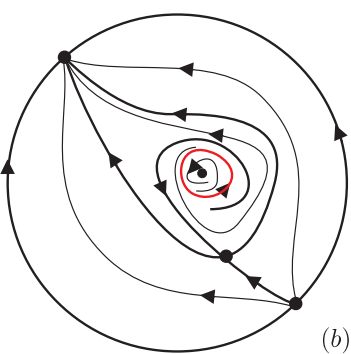

(b)
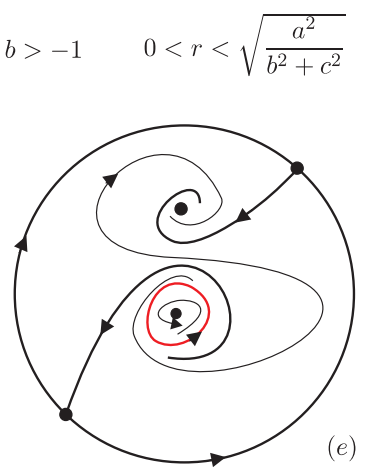

(e)

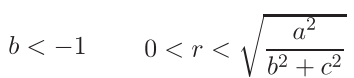

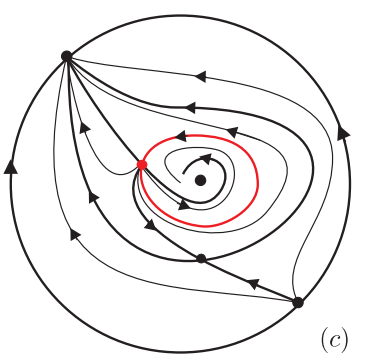
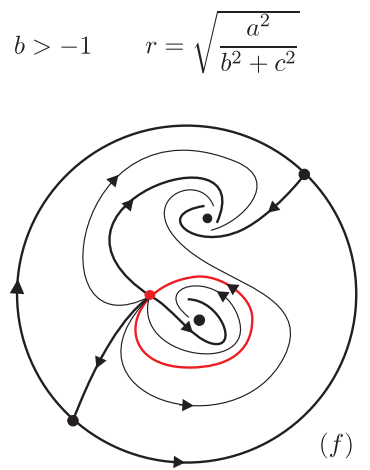

$b<-1 \quad r=\sqrt{\frac{a^{2}}{b^{2}+c^{2}}}$

FiguRE 1. Birth and death of the algebraic limit cycles of degree 2. 
Theorem 1. (Degree 2) Each planar quadratic system having an invariant ellipse after an affine change of variables and a rescaling of the time, can be written as

$$
\begin{aligned}
& \dot{x}=r^{2}-x^{2}-y^{2}-y(c+a x+b y), \\
& \dot{y}=x(c+a x+b y),
\end{aligned}
$$

where $r>0$ and $a^{2}+b^{2}+c^{2} \neq 0$. The invariant ellipse is

$$
x^{2}+y^{2}-r^{2}=0
$$

For $\left(a^{2}+b^{2}\right) r^{2}-c^{2}<0, c^{2}+4(1+b) r^{2}>0, b \neq-1$ and $a \neq 0$ this ellipse is an algebraic limit cycle of degree 2, see Figure $1(b)$ and $(e)$, for $b>-1$ and $b<-1$, respectively. Moreover, the family of these limit cycles borns or dies at a Hopf bifurcation when $r$ goes to zero, see Figure $1(a)$ and $(d)$ for $b>-1$ and $b<-1$, respectively; or in a homoclinic orbit formed by an ellipse having a saddle-node on it when $\left(a^{2}+b^{2}\right) r^{2}-c^{2}=0$, see Figure $1(c)$ and $(f)$ for $b>-1$ and $b<-1$, respectively. We note this algebraic limit cycle can be stable or unstable depending on the values of the parameters. Here we only drawn the phase portraits in the case that such a limit cycle is unstable.

Theorem 2. (Degree 4) We have the following four families of algebraic limit cycles of degree 4 in quadratic systems, modulo an affine change of variables and a rescaling of the time.

(a) The Yablonskii's system

$\dot{x}=-4 a b c x+3(a+b) c x^{2}-(a+b) y+4 x y$,

$\dot{y}=a b(a+b) x+\left(4 a b-\frac{3}{2}(a+b)^{2}+4 a b c^{2}\right) x^{2}-4 a b c y+8(a+b) c x y+8 y^{2}$,

has the irreducible invariant algebraic curve of degree four

$$
x^{2}(x-a)(x-b)+\left(c x^{2}+y\right)^{2}=0 .
$$

For $a b c \neq 0, a \neq b, a b>0$ and $4 c^{2}(a-b)^{2}+(3 a-b)(a-3 b)<0$ this curve contains an algebraic limit cycle of degree 4, see Figure 2(a). This family of limit cycles borns or dies at a Hopf bifurcation when either $a=b$, or $a=0$, or $b=0$, see Figure 2(b); or from a homoclinic orbit formed by an algebraic oval and a saddlenode on it when $4 c^{2}(a-b)^{2}+(3 a-b)(a-3 b)=0$, see Figure $2(c)$; or from $a$ periodic orbit of a center when $c=0$, see Figure $2(d)$. We note this algebraic limit cycle can be stable or unstable depending on the values of the parameters. Here we only drawn the phase portraits in the case that such a limit cycle is unstable.

(b) The Filiptsov's system

$$
\begin{aligned}
& \dot{x}=6(1+a) x-6(2+a) x^{2}+2 y+12 x y, \\
& \dot{y}=3 a(1+a) x^{2}+15(1+a) y-2(9+5 a) x y+16 y^{2},
\end{aligned}
$$

has the irreducible invariant algebraic curve

$$
3(1+a)\left(a x^{2}+y\right)^{2}+2 y^{2}(2 y-3(1+a) x)=0 .
$$


For $0<a<3 / 13$ this curve contains an unstable algebraic limit cycle of degree 4 as the one shown in Figure 3(b). When a $\searrow 0$ this family of limit cycles tends to a heteroclinic cycle having some orbit at infinity, see Figure $3(a)$. When a $\nearrow 3 / 13$ the family of limit cycles borns or died in a Hopf bifurcation, see Figure $3(c)$.

(c) The Chavarriga's system

$$
\begin{aligned}
& \dot{x}=5 x+6 x^{2}+4(1+a) x y+a y^{2}, \\
& \dot{y}=x+2 y+4 x y+(2+3 a) y^{2},
\end{aligned}
$$

has the irreducible invariant algebraic curve

$$
x^{2}+x^{3}+x^{2} y+2 a x y^{2}+2 a x y^{3}+a^{2} y^{4}=0 .
$$

When $(17 \sqrt{17}-71) / 32<a<0$ this curve contains a stable algebraic limit cycle of degree 4 , see Figure $4(b)$. When a $\searrow(17 \sqrt{17}-71) / 32$ this family of limit cycles borns or dies at a Hopf bifurcation, see Figure 4(a); when a $\nearrow 0$ this family of limit cycles borns or dies in a heteroclinic loop having some orbit at infinity, see Figure 4(c).

(d) The Chavarriga, Llibre and Sorolla's system

$$
\begin{aligned}
& \dot{x}=2\left(1+2 x-2 a x^{2}+6 x y\right), \\
& \dot{y}=8-3 a-14 a x-2 a x y-8 y^{2} .
\end{aligned}
$$

This system has the irreducible invariant algebraic curve

$$
\frac{1}{4}+x-x^{2}+a x^{3}+x y+x^{2} y^{2}=0 .
$$

When $0<a<1 / 4$ this curve contains an unstable algebraic limit cycle of degree 4, see Figure 5(b). When a $\searrow 0$ the equilibrium point $p_{+}$tends to the infinite equilibrium $(0,0)$ of $U_{1}$, and the limit cycle tends to an orbit of the elliptic sector of that infinite equilibrium. Moreover, the equilibrium $q_{-}$tends to the infinite equilibrium $(0,0)$ of $V_{1}$ (see the definitions of the equilibria $p_{+}$and $q_{-}$in the proof of this statement), see Figure $5(a)$. When a $\nearrow 1 / 4$ this family of limit cycles borns or dies at a Hopf bifurcation, see Figure $5(c)$.

We remark that in statement $(d)$ of Theorem 2 we have shown the existence of a limit cycle bifurcating from an orbit of an elliptic sector of an infinite equilibrium point. This kind of bifurcation of limit cycles as far as we know untill now was very unusual. This phenomenon also will happens for a family of algebraic limit cycles of degree 5 as we shall see in the next theorem.

In [6] the authors gave two one-parameter families of quadratic system with algebraic limit cycles of degree 5 and 6 . Recently in [1] the authors present a new one-parameter family of quadratic systems with a new algebraic limit cycle of degree 5 .

Theorem 3. (Degree 5) We have the following two one-parameter families of algebraic limit cycles of degree 5 in quadratic systems. 

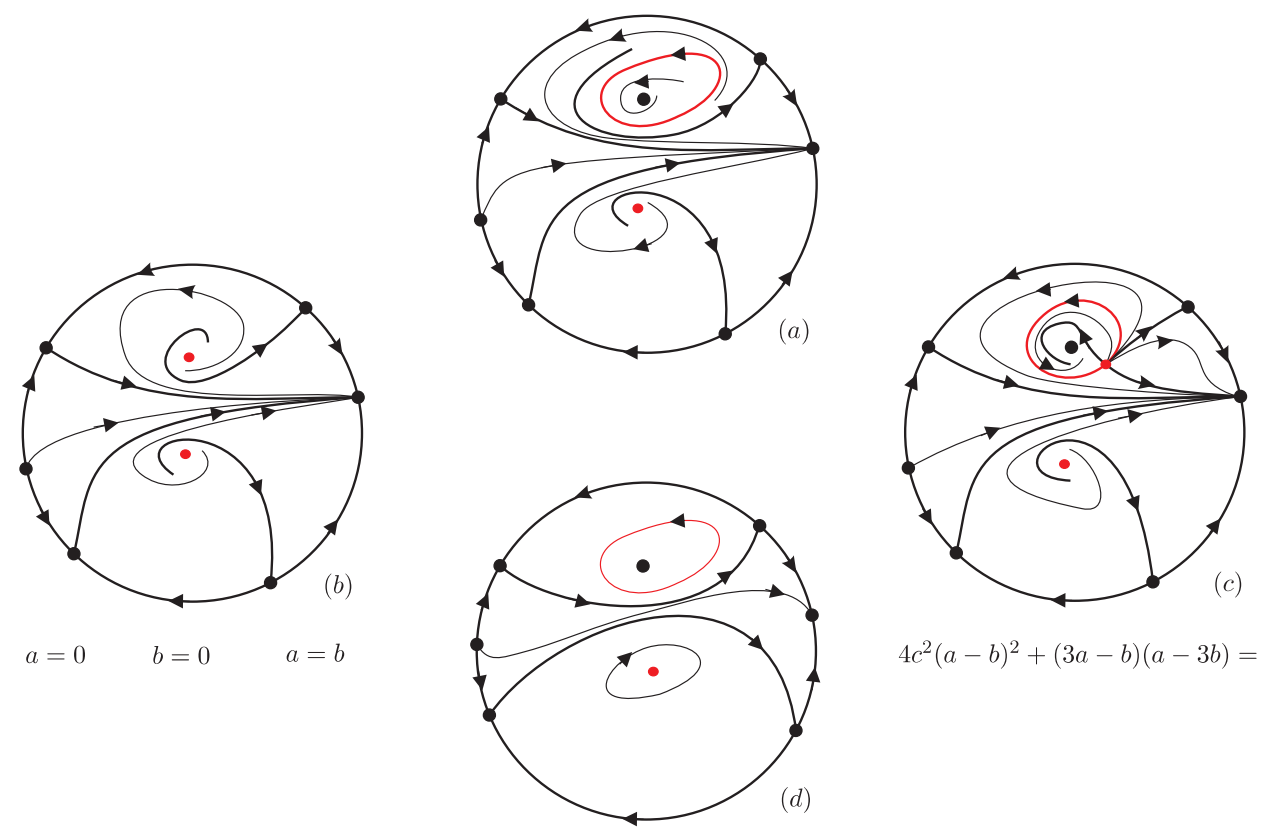

$4 c^{2}(a-b)^{2}+(3 a-b)(a-3 b)=0$

$c=0$

FiguRE 2. Birth and death of the algebraic limit cycles of the Yablonskii's system.
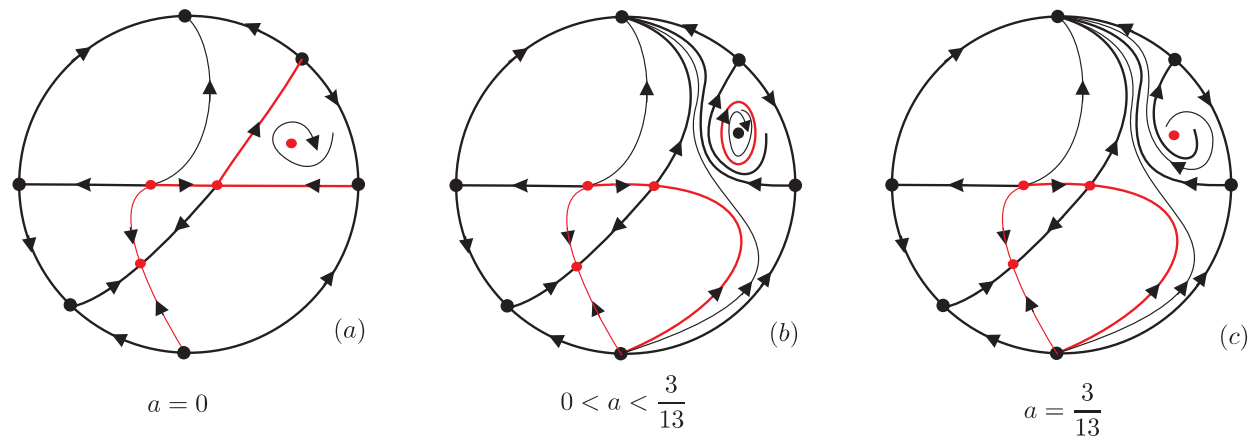

Figure 3. Birth and death of the algebraic limit cycles of the Filiptsov's system.

(a) The Christopher, Llibre and Swirszcz's system

$$
\begin{aligned}
& \dot{x}=28 x-12 /(a+4) y^{2}-2\left(a^{2}-16\right)(12+a) x^{2}+6(3 a-4) x y \\
& \dot{y}=\left(32-2 a^{2}\right) x+8 y-(a+12)\left(a^{2}-16\right) x y+(10 a-24) y^{2}
\end{aligned}
$$




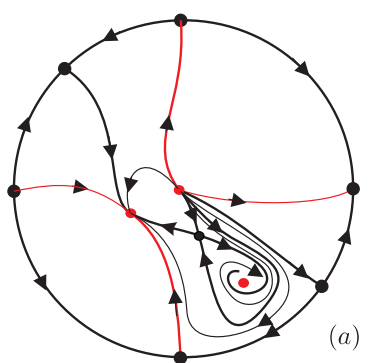

$a=\frac{17 \sqrt{17}-71}{32}$

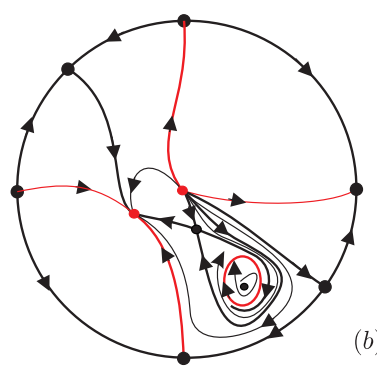

$\frac{17 \sqrt{17}-71}{32}<a<0$

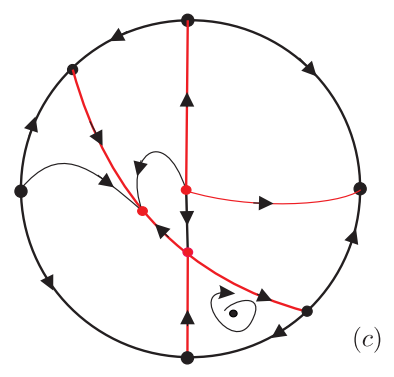

$a=0$

Figure 4. Birth and death of the algebraic limit cycles of the Chavarriga's system.
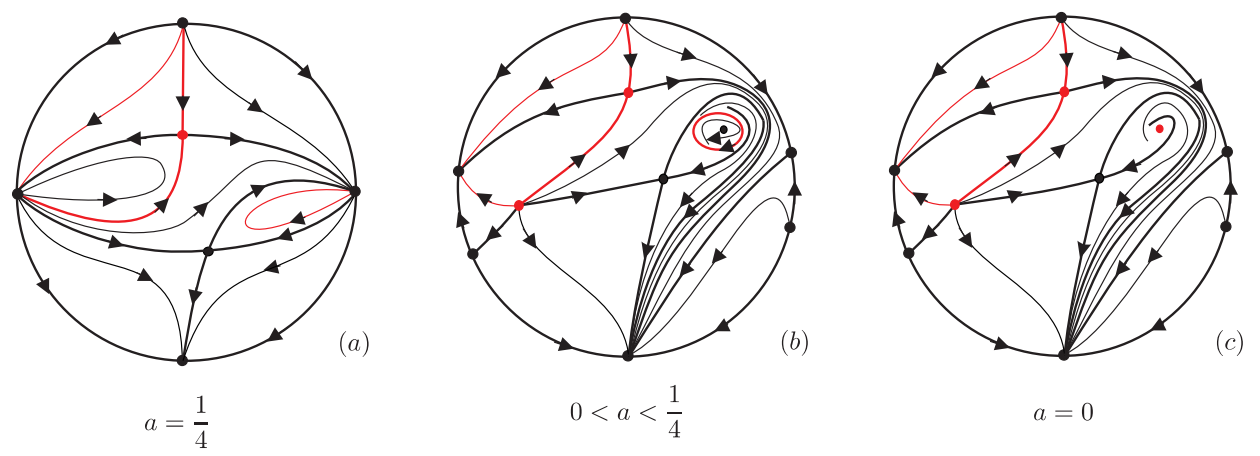

FiguRE 5. Birth and death of the algebraic limit cycles of the Chavarriga, Llibre and Sorolla's system.
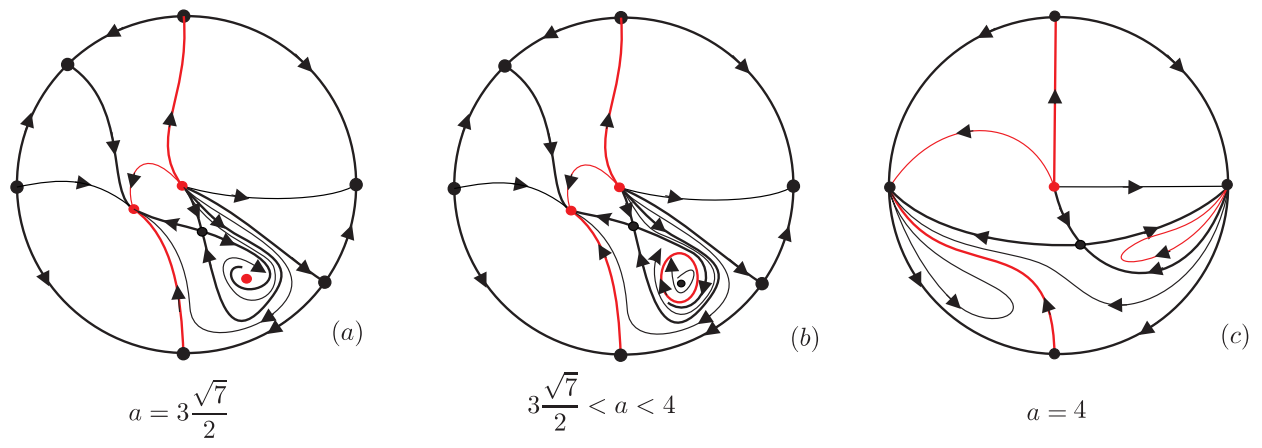

Figure 6. Birth and death of the algebraic limit cycles of the Christopher, Llibre and Swirszcz's system of degree 5.

has the irreducible invariant algebraic curve

$$
\begin{aligned}
& x^{2}+\left(16-a^{2}\right) x^{3}+(a-2) x^{2} y+\frac{1}{(4+a)^{2}} y^{4}-\frac{6}{(4+a)^{2}} y^{5}-\frac{2}{4+a} x y^{2}+ \\
& \frac{(a-4)(12+a)}{4} x^{2} y^{2}+\frac{(12+a)}{4+a} x y^{4}+\frac{8-a}{4+a} x y^{3}=0 .
\end{aligned}
$$


When $a \in(3 \sqrt{7} / 2,4)$, this curve contains a stable algebraic limit cycle of degree 5 , see Figure $6(b)$. When a $\searrow 3 \sqrt{7} / 2$ this family of limit cycles borns or dies at a Hopf bifurcation, see Figure $6(a)$; and when a $\nearrow 4$ the equilibrium point $p_{1}$ tends to the infinite equilibrium $(0,0)$ of $U_{1}$, the limit cycle tends to an orbit of the elliptic sector of that infinite equilibrium, and the equilibrium $p_{+}$tends to the equilibrium $(0,0)$ of $V_{1}$ (see the definitions of the equilibria $p_{1}$ and $p_{+}$in the proof of this statement), see Figure 6(c).

(b) The Alberich-Carramiñana, Ferragut and Llibre's system

(13)

$\dot{x}=2(a+12)\left(168 a^{2} x+3 y\right)-16 a\left(28 a^{2}+9 a \gamma-126 a-18 \gamma-252\right) x^{2}-7 a-3 \gamma+42 x y$,

$\dot{y}=(a+12)\left(-1344 a^{3}(a-30) x-72 a(a-2) y\right)-\left(64 a^{2}\left(476 a^{3}+3 a^{2}(61 \gamma+910)+\right.\right.$

$288 a(3 \gamma+77)-108 \gamma+28728) x^{2}-12 a\left(77 a^{2}+27 a \gamma+30 \gamma+1260\right) x y-(7 a+3 \gamma-42) y^{2}$,

with $\gamma=\sqrt{7\left(108+a^{2}\right)}$ has the irreducible invariant algebraic curve of degree five

$6912(a-2)^{2} a^{3}(a+12)^{6}(2 a+3)-\left(13824(a-2)^{2} a^{3}(a+12)^{5}(2 a+3)(-24+5 a)+\right.$

$\left.20736(a-2)^{2} a^{3}(a+12)^{5}(3+2 a) \gamma\right) x-864(a-2)^{2} a^{2}(a+12)^{5}(2 a+3) y$

$+\left(145152(-6+a)(a-2)^{2} a^{3}(a+12)^{4}(2 a+3) \gamma+1152(a-2)^{2} a^{3}(a+12)^{4}(63288+28044 a\right.$

$\left.\left.-3774 a^{2}+685 a^{3}\right)\right) x^{2}+\left(1728(a-2)^{2} a^{2}(a+12)^{4}(2 a+3) \gamma+288(a-2)^{2} a^{2}(a+12)^{4}\right.$

$\left.\left(-468-36 a+37 a^{2}\right)\right) x y+54(a-2)^{2} a(a+12)^{5} y^{2}+\left(-6912(a-9)(a-2)^{2} a^{3}(a+12)^{3} \gamma\right.$

$\left(-1188-408 a+109 a^{2}\right)-1792 a^{3}(a+12)^{3}\left(-4999968+4201632 a+1662552 a^{2}-950004 a^{3}\right.$

$\left.\left.+148836 a^{4}-18865 a^{5}+1116 a^{6}\right)\right) x^{3}+\left(-432(a-2)^{2} a^{2}(a+12)^{3} \gamma\left(-612-144 a+29 a^{2}\right)\right.$

$\left.-336 a^{2}(a+12)^{3}\left(59616-134784 a-32688 a^{2}+5580 a^{3}-902 a^{4}+99 a^{5}\right)\right) x^{2} y$

$+\left(-54(a-2)^{2} a(a+12)^{4} \gamma-126(a-2) a(a+12)^{4}\left(108+a^{2}\right)\right) x y^{2}-14(a+12)^{5} y^{3}$

$+\left(2688(a-30) a^{3}(a+12)^{2} \gamma\left(71280-110808 a-43272 a^{2}+5310 a^{3}-2153 a^{4}+252 a^{5}\right)\right.$

$+1344(a-30) a^{3}(a+12)^{2}\left(-5520960-1314144 a-948456 a^{2}-823284 a^{3}+99942 a^{4}\right.$

$\left.\left.-11421 a^{5}+1334 a^{6}\right)\right) x^{4}+\left(336(a-30) a^{2}(a+12)^{2} \gamma\left(-4320+3312 a+1848 a^{2}+82 a^{3}+39 a^{4}\right)\right.$

$\left.+336(a-30) a^{2}(a+12)^{2}\left(25920+57888 a+18504 a^{2}+10596 a^{3}+222 a^{4}+103 a^{5}\right)\right) x^{3} y$

$+\left(21(a-30) a(a+12)^{3} \gamma\left(60+20 a+3 a^{2}\right)+42(a-30) a(a+12)^{3}(900+264 a\right.$

$\left.\left.+27 a^{2}+4 a^{3}\right)\right) x^{2} y^{2}+\left(28224(a-30) a^{3}(a+12) \gamma\left(139968+194400 a+101448 a^{2}+30420 a^{3}\right.\right.$

$\left.+2258 a^{4}+477 a^{5}\right)+56448(a-30) a^{3}(a+12)\left(824256+3304800 a+1523232 a^{2}+294840 a^{3}\right.$ 


$$
\begin{aligned}
& \left.\left.+74304 a^{4}+2988 a^{5}+631 a^{6}\right)\right) x^{5}+\left(1 4 1 1 2 ( a - 3 0 ) a ^ { 2 } ( a + 1 2 ) \gamma \left(3240+2268 a+642 a^{2}\right.\right. \\
& \left.+55 a^{3}+12 a^{4}\right)+3528(a-30) a^{2}(a+12)\left(440640+222048 a+55512 a^{2}+13644 a^{3}\right. \\
& \left.\left.+582 a^{4}+127 a^{5}\right)\right) x^{4} y=0 .
\end{aligned}
$$

When $a \in(3 / 2,2)$ this curve contains an unstable algebraic limit cycle of degree 5 , see Figure 7(b). When a $\searrow 3 / 2$ this family of limit cycles borns or dies at a Hopf bifurcation, see Figure $7(a)$; and when a $\nearrow 2$ this family of limit cycles borns or dies at a heteroclinic loop formed by two pieces, one of a parabola and the other a straight line, and two saddles, see Figure $7(c)$.
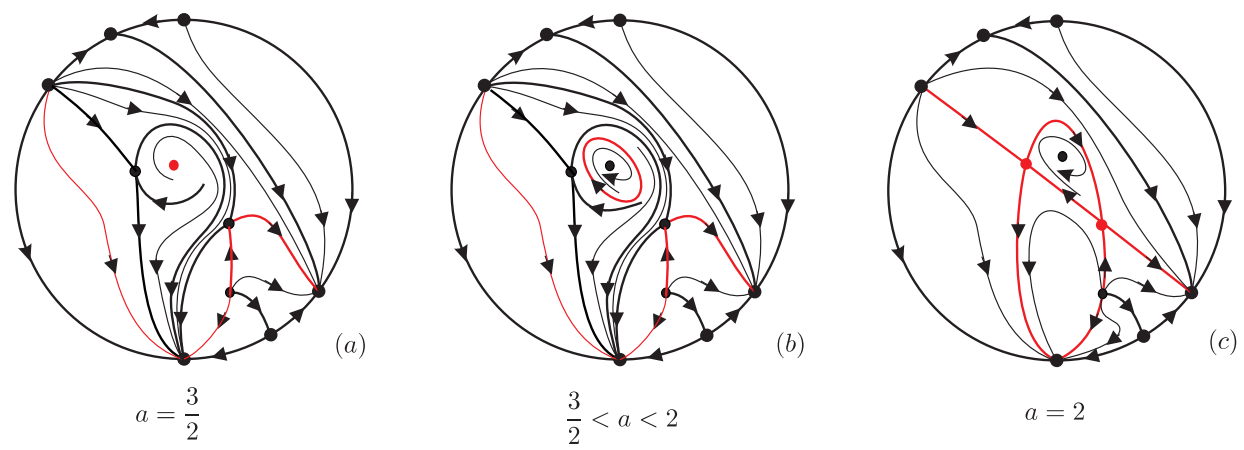

FiguRE 7. Birth and death of the algebraic limit cycles of a system of the Alberich-Carramiñana, Ferragut and Llibre's system.

Theorem 4. (Degree 6) The unique known family of algebraic limit cycle of degree 6 in quadratic systems is due to Christopher, Llibre and Swirszcz, and it is given by the system

$$
\begin{aligned}
& \dot{x}=28(a-30) a x+y+168 a^{2} x^{2}+3 x y, \\
& \dot{y}=16 a(a-30)\left(14(a-30) a x+5 y+84 a^{2} x^{2}\right)+24(17 a-6) a x y+6 y^{2} .
\end{aligned}
$$

This system has the one-parameter family of irreducible algebraic invariant curves of degree 6

$$
\begin{aligned}
& -7 y^{3}+3(a-30)^{2} a y^{2}+18(a-30)(-2+a) a x y^{2}+27(a-2)^{2} a x^{2} y^{2}+ \\
& 24(a-30)^{3} a^{2} x y+144(a-30)(a-2)^{2} a^{2} x^{3} y+48(a-30)^{4} a^{3} x^{2}+ \\
& 576(a-30)^{2}(-2+a)^{2} a^{3} x^{4}-432(a-2)^{2} a^{2}(3+2 a) x^{4} y- \\
& 3456(a-30)(-2+a)^{2} a^{3}(3+2 a) x^{5}+3456(a-2)^{2} a^{3}(12+a)(3+2 a) x^{6}+ \\
& 24(a-30)^{2} a^{2}(9 a-4) x^{2} y+64(a-30)^{3} a^{3}(9 a-4) x^{3}=0 .
\end{aligned}
$$

When $a \in(3 / 2,2)$ this curve contains a stable algebraic limit cycle of degree 6 , see Figure $8(b)$. When a \3/2 this family of limit cycles borns or dies at a Hopf bifurcation, see Figure $8(a)$; and when a $\nearrow 2$ this family of limit cycles borns or dies in a heteroclinic loop having some orbits at infinity, see Figure 8(c).

The proof of Theorems 1, 2, 3 and 4 are in sections $3,4,5$ and 6 , respectively. 

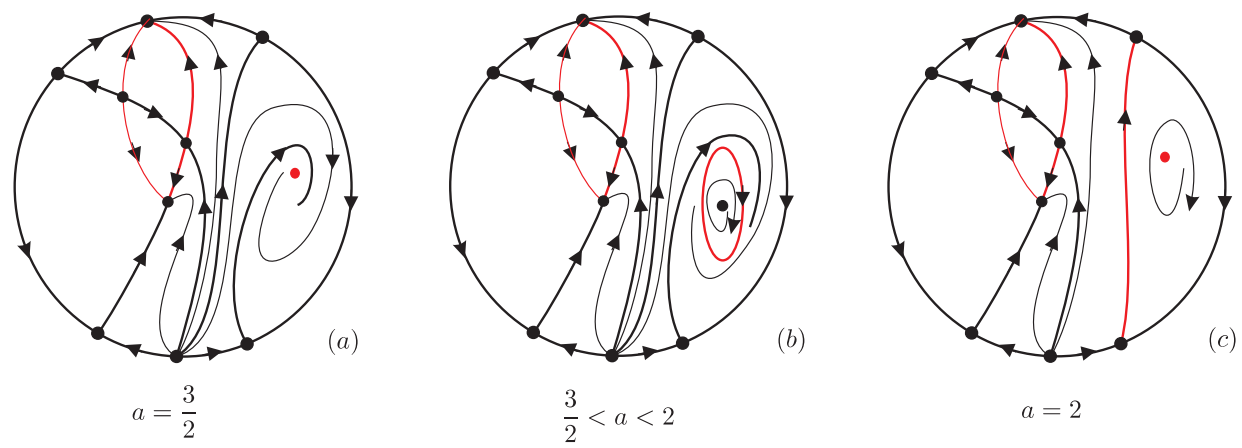

Figure 8. Birth and death of the algebraic limit cycles of the Christopher, Llibre and Swirszcz's system of degree 6 .

\section{Preliminaries}

An equilibrium point $p$ such that the eigenvalues of the linear part of the differential system at $p$ have non-zero real part is called hyperbolic equilibrium. The local phase portraits of the hyperbolic equilibria are determined by their eigenvalues, see for instance Theorem 2.15 of [7]. If only one of the two eigenvalues of an equilibrium is zero, then we say that this is a semi-hyperbolic equilibrium, and the local phase portraits of such equilibria are well known, see for example Theorem 2.19 of [7].

In this paper all the phase portraits are drawn in the so called Poincaré disc, which is roughly speaking, the closed disc centered at the origin of the coordinates and of radius one. The interior of this disc is identified with $\mathbb{R}^{2}$. Its boundary, the circle $\mathbb{S}^{1}$, is identified with the infinity of $\mathbb{R}^{2}$. In the plane $\mathbb{R}^{2}$ we can go to infinity in as many directions as points in the circle $\mathbb{S}^{1}$. For more details about the Poincaré disc, see Chapter 5 of [8].
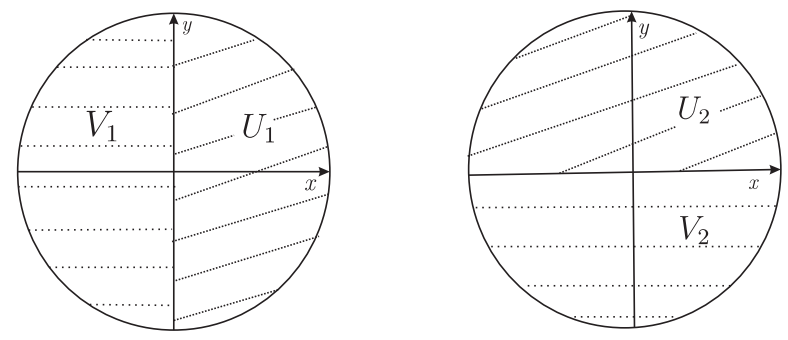

Figure 9. The local charts $U_{i}$ and $V_{i}$, for $i=1,2$ of the Poincaré disc $\mathbb{D}$.

In order to work with the Poincaré disc we need four local charts. They are represented in Figure 1 and they are defined as

$$
\begin{array}{ll}
U_{1}=\left\{(x, y) \in \mathbb{R}^{2}: x>0\right\}, & V_{1}=\left\{(x, y) \in \mathbb{R}^{2}: x<0\right\}, \\
U_{2}=\left\{(x, y) \in \mathbb{R}^{2}: y>0\right\}, & V_{2}=\left\{(x, y) \in \mathbb{R}^{2}: y<0\right\} .
\end{array}
$$



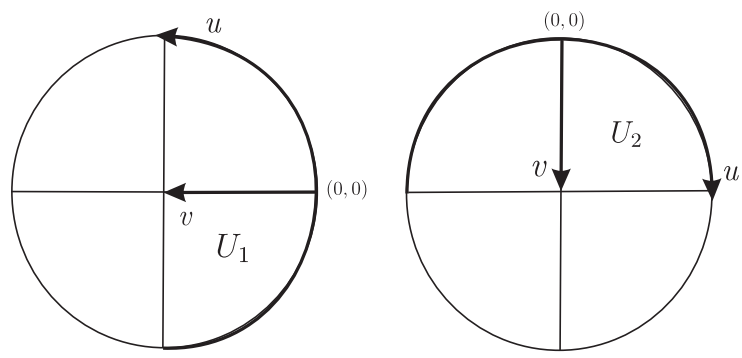

Figure 10. The coordinates $(u, v)$ in the local charts $U_{1}$ and $U_{2}$.

We define $\phi_{k}: U_{k} \rightarrow \mathbb{D}$ and $\psi_{k}: V_{k} \rightarrow \mathbb{D}$ for $k=1,2$ as follows

$$
\phi_{1}(x, y)=\left(\frac{y}{x}, \frac{1}{x}\right)=(u, v), \quad \phi_{2}(x, y)=\left(\frac{1}{y}, \frac{x}{y}\right)=(u, v),
$$

and $\psi_{k}=-\phi_{k}, k=1,2$. In Figure 2 this local coordinates are represented in the local charts $U_{1}$ and $U_{2}$, respectively.

If $\dot{x}=P(x, y), \dot{y}=Q(x, y)$ is a polynomial differential system and $d$ is the maximum of the degrees of $P$ and $Q$, then the expression of the extended flow on the local chart $\left(U_{1}, \phi_{1}\right)$ is

$$
\dot{u}=v^{d}\left[-u P\left(\frac{y}{x}, \frac{1}{x}\right)+Q\left(\frac{y}{x}, \frac{1}{x}\right)\right], \quad \dot{v}=-v^{d+1} P\left(\frac{y}{x}, \frac{1}{x}\right) .
$$

The expression on the local chart $\left(U_{2}, \phi_{2}\right)$ is

$$
\dot{u}=v^{d}\left[P\left(\frac{1}{y}, \frac{x}{y}\right)-u Q\left(\frac{1}{y}, \frac{x}{y}\right)\right], \quad \dot{v}=-v^{d+1} Q\left(\frac{1}{y}, \frac{x}{y}\right) .
$$

The expressions in the local charts $\left(V_{k}, \psi_{k}\right)$, for $k=1,2$ are the same than in the chart $\left(U_{k}, \phi_{k}\right)$ multiplied by $(-1)^{d-1}$. We note that $v=0$ corresponds to the infinity $\mathbb{S}^{1}$ in all the local charts.

Let $\mathcal{X}_{1}$ and $\mathcal{X}_{2}$ be two polynomial vector fields on $\mathbb{R}^{2}$, and let $p\left(\mathcal{X}_{1}\right)$ and $p\left(\mathcal{X}_{2}\right)$ be their respective polynomial vector fields on the Poincaré disc $\mathbb{D}$. We say that they are topologically equivalent if there exists a homeomorphism on the Poincaré disc $\mathbb{D}$ which preserves the infinity $\mathbb{S}^{1}$ and sends the orbits of $p\left(\mathcal{X}_{1}\right)$ to orbits of $p\left(\mathcal{X}_{2}\right)$, preserving or reversing the orientation of all the orbits.

A separatrix of the Poincaré compactification $p(\mathcal{X})$ is one of following orbits: the finite equilibrium point, the limit cycles, all the orbits at the infinity $\mathbb{S}^{1}$, and the two orbits at the boundary of a hyperbolic sector at a finite or an infinite equilibrium point, see for more details on the separatrices [20,21]. 
The set of all separatrices of $p(\mathcal{X})$, denote by $\Sigma_{\mathcal{X}}$, is a closed set (see [21]). A canonical region of $p(\mathcal{X})$ is an open connected component of $\mathbb{D} \backslash \Sigma_{\mathcal{X}}$.

A separatrix configuration of $p(\mathcal{X})$, denoted by $\Sigma_{\mathcal{X}}^{\prime}$ is the union of the set $\Sigma_{\mathcal{X}}$ with an orbit of each canonical region. Two separatrix configurations $\Sigma_{\mathcal{X}_{1}}^{\prime}$ and $\Sigma_{\mathcal{X}_{2}}^{\prime}$ are topologically equivalent if there is a homeomorphism $h: \mathbb{D} \longrightarrow \mathbb{D}$ such that $h\left(\Sigma_{\mathcal{X}_{1}}^{\prime}\right)=\Sigma_{\mathcal{X}_{2}}^{\prime}$.

According to the following theorem which was proved by Markus [20], Neumann [21] and Peixoto [22], it is sufficient to investigate the separatrix configuration of a polynomial differential system, for determining its global phase portrait.

Theorem 5. Two Poincaré compactified polynomial vector fields $p\left(\mathcal{X}_{1}\right)$ and $p\left(\mathcal{X}_{2}\right)$ with finitely many separatrices are topologically equivalent if and only if their separatrix configurations $\Sigma_{\mathcal{X}_{1}}^{\prime}$ and $\Sigma_{\mathcal{X}_{2}}^{\prime}$ are topologically equivalent.

\section{Proof of Theorem 1}

The quadratic system (2) has the following equilibria

$$
\begin{aligned}
& p_{ \pm}=\left(0,-\frac{c \pm \sqrt{c^{2}+4(1+b) r^{2}}}{2(1+b)}\right) \\
& q_{ \pm}=\left(\frac{ \pm b \sqrt{a^{2}\left(r^{2}\left(a^{2}+b^{2}\right)-c^{2}\right)}-a^{2} c}{a\left(a^{2}+b^{2}\right)}, \frac{\mp \sqrt{a^{2}\left(r^{2}\left(a^{2}+b^{2}\right)-c^{2}\right)}-b c}{a^{2}+b^{2}}\right) .
\end{aligned}
$$

The quadratic system (2) has the invariant ellipse $x^{2}+y^{2}-r^{2}=0$ for $0<r<\sqrt{\frac{c^{2}}{a^{2}+b^{2}}}$ as a limit cycle because the unique equilibria on the ellipse are $q_{ \pm}$which for these values of $r$ are complex (i.e. their coordinates are not real), so the invariant ellipse is a periodic orbit, but since one of the points $p_{ \pm}$is inside the region limited by the circle $x^{2}+y^{2}-r^{2}=0$ and it is a focus the invariant circle is a limit cycle.

The fact that under the assumption of Theorem 1 the invariant ellipse $x^{2}+y^{2}-r^{2}=0$ is an algebraic limit cycle was proved in [24], see also [3, 4, 19].

When $r$ goes to zero the circle tends to an equilibrium point, the focus. So the limit cycle on the circle borns or dies in a Hopf bifurcation. On the other hand when $r=\sqrt{\frac{c^{2}}{a^{2}+b^{2}}}$ the complex equilibria $q_{ \pm}$becomes a saddle-node on the circle. In other words, the limit cycle on the circle ends in a homoclinic orbit to this saddle-node. See Figure 1.

Now we study the infinite equilibrium point in the Poincaré disc. System (2) in the local chart $U_{1}$ writes

$$
\begin{aligned}
& \dot{u}=a+(1+b) u+c v+a u^{2}+(1+b) u^{3}+c u^{2} v-r^{2} u v^{2}, \\
& \dot{v}=v\left(1+a u+(1+b) u^{2}+c u v-r^{2} v^{2}\right),
\end{aligned}
$$


and in the local chart $U_{2}$ is

$$
\begin{aligned}
& \dot{u}=-(1+b)-a u-c v-(1+b) u^{2}+r^{2} v^{2}-a u^{3}-c u^{2} v \\
& \dot{v}=-v\left(-(1+b)-a u-c v-u^{2}+r^{2} v^{2}\right)
\end{aligned}
$$

If $b \neq-1$ the unique infinite equilibrium point in $U_{1}$ is $(-a /(1+b), 0)$, which is a hyperbolic unstable node, and the origin of $U_{2}$ is not an infinite equilibrium point. If $b=-1$ there is no infinite equilibrium point in $U_{1}$, but then the origin of the local chart $U_{2}$ becomes a hyperbolic unstable node.

In summary, knowing the local phase portraits in all finite and infinite equilibria, together with the information about the invariant circle when $0 \leq r \leq \sqrt{\frac{c^{2}}{a^{2}+b^{2}}}$ we obtain the phase portraits in the Poincaré disc of system (2) described in Figure 1. This completes the proof of Theorem 1.

\section{Proof of Theorem 2}

The existence of the families of algebraic limit cycles of degree 4 under the conditions of the different statements of Theorem 2 where proved in $[3,4,12,24]$.

4.1. Proof of statement (a) of Theorem 2. The quadratic system (3) has the following equilibria

$$
\begin{aligned}
& p_{0}=(0,0), \quad p_{1}=\left(\frac{2 a b(a+b)}{3 a^{2}-2 a b+3 b^{2}},-\frac{4 a^{2} b^{2} c}{3 a^{2}-2 a b+3 b^{2}}\right), \\
& p_{ \pm}=\left(\frac{2 c^{2}(a+b)+a+b \pm R}{4\left(c^{2}+1\right)},-\frac{\left(2 c^{2}(a+b) \pm R\right)\left(2 c^{2}(a+b)+a+b \pm R\right)}{16 c\left(c^{2}+1\right)}\right),
\end{aligned}
$$

where $R=\sqrt{c^{2}\left(4 c^{2}(a-b)^{2}+(3 a-b)(a-3 b)\right)}$. Note that the equilibria $p_{0}$ and $p_{1}$ always exist, while the points $p_{ \pm}$are complex if $4 c^{2}(a-b)^{2}+(3 a-b)(a-3 b)<0$.

Under the assumptions of statement $(a)$ of Theorem 2 the invariant curve (4) is formed by an oval $O$ surrounding the equilibrium $p_{1}$, and an isolated equilibrium $p_{0}$. So the oval $O$ is a periodic orbit and since $p_{1}$ is a hyperbolic stable focus the oval $O$ is an unstable limit cycle, see Figure 2(a).

When either $a \rightarrow 0$, or $b \rightarrow 0$, or $a \rightarrow b$ the oval $O$ tends to the focus $p_{1}$. Therefore when either $a=0$, or $b=0$, or $a=b$ we have a Hopf bifurcation where borns or dies the limit cycle $O$, see Figure 2(b).

When $4 c^{2}(a-b)^{2}+(3 a-b)(a-3 b) \nearrow 0$, then complex equilibria $p_{ \pm}$become a saddlenode on the oval $O$, so the limit cycle $O$ ends in a homoclinic loop at this saddle-node, see Figure 2(c).

Finally when $c \rightarrow 0$ the point $p_{1}$ tends to a center and the oval $O$ tends to a periodic orbit surrounding the center, see Figure $2(d)$. This fact was proved in [18]. 
In order to complete the phase portrait in the Poincaré disc we must to study the infinite equilibria. In the local chart $U_{1}$ system (3) becomes

$$
\begin{aligned}
& \dot{u}=4 a b-3 / 2(a+b)^{2}+4 a b c^{2}+5(a+b) c u+a b(a+b) v+4 u^{2}+(a+b) u^{2} v, \\
& \dot{v}=v(-3 c(a+b)-4 u+4 a b c v+(a+b) u v),
\end{aligned}
$$

and in the local chart $U_{2}$ is

$$
\begin{aligned}
& \dot{u}=\frac{-8 u-2(a+b) v-10(a+b) c u^{2}+\left(3 a^{2}-2 a b+3 b^{2}-8 a b c^{2}\right) u^{3}-2 a b(a+b) u^{2} v}{2}, \\
& \dot{v}=-v\left(4 u-(a+b) v+3(a+b) c u^{2}-4 a b c u v\right) .
\end{aligned}
$$

Denote by $S=\sqrt{-2 a b\left(8+7 c^{2}\right)+a^{2}\left(24+25 c^{2}\right)+b^{2}\left(24+25 c^{2}\right)}$. The expression $S$ is always real. Then the infinite equilibria in $U_{1}$ are $q_{ \pm}=((-5 c(a+b) \pm S) / 8,0)$. Note that $-5 c(a+b)-S<-5 c(a+b)+S$ and that $q_{-}$is a hyperbolic saddle, and $q_{+}$is a hyperbolic stable node.

The origin of $U_{2}$ is always a semi-hyperbolic infinite equilibrium with eigenvalues -4 and 0. Applying Theorem 2.19 of [7] this equilibrium is a saddle.

Taking into account the local phase portraits of the finite and infinite equilibria together with the invariant algebraic curve and the previous information it follows the phase portraits in the Poincaré disc of system (3) described in Figure 2. This proves statement (a).

4.2. Proof of statement (b) of Theorem 2. The quadratic system (5) when $a \in$ $[0,3 / 13]$ has the following four equilibria

$$
\begin{aligned}
& p_{0}=(0,0), \quad p_{1}=\left(\frac{5(a+1)}{2(1-a)}, \frac{15(a+1)^{2}}{8(1-a)}\right), \\
& p_{ \pm}=\left(\frac{a-R}{12(a+1)},-\frac{a(16 a+2 R+33)+3(R+6)}{24(a+1)}\right),
\end{aligned}
$$

where $R=\sqrt{36+a(72+37 a)}$.

For $a \in(0,3 / 13)$ the invariant algebraic curve (6) is formed by an oval $O$ surrounding the hyperbolic stable focus $p_{1}$, and a component homeomorphic to a straight line ending at the infinite equilibrium localized at the origin of $V_{2}$ which contains the finite equilibria $p_{0}$ and $p_{ \pm}$, where $p_{0}$ is a hyperbolic unstable node, $p_{+}$is a hyperbolic saddle, and $p_{-}$is a hyperbolic stable node. So the oval $O$ is an unstable limite cycle, see Figure 3(b).

When $a \searrow 0$ the oval $O$ increases tending to a heteroclinic loop with an orbit contained at infinity, see Figure 3(a). When $a \nearrow 3 / 13$ the oval $O$ tends to the equilibrium point $p_{1}$, see Figure 3(c), and consequently for $a=3 / 13$ we have a Hopf bifurcation.

In the local chart $U_{1}$ system (5) becomes

$$
\begin{aligned}
& \dot{u}=3 a(1+a)-2 u(3+2 a)+4 u^{2}+9(1+a) u v-2 u^{2} v, \\
& \dot{v}=2 v(3(2+a)-6 u-3(1+a) v-u v),
\end{aligned}
$$


and in the local chart $U_{2}$ is

$$
\begin{aligned}
& \dot{u}=-4 u+2 v+2(3+2 a) u^{2}-9(1+a) u v-3 a(1+a) u^{3}, \\
& \dot{v}=2 v\left(-6 u-v+3(2+a) u^{2}-3(1+a) u v\right) .
\end{aligned}
$$

In $U_{1}$ we have the two equilibria $q_{ \pm}=\left(\left(3+2 a \pm \sqrt{9-8 a^{2}}\right) / 4,0\right)$, both are hyperbolic saddles. The origin of the local chart $U_{2}$ is a hyperbolic stable node.

Taking into account the previous information it follows the three phase portraits in the Poincaré disc of system (5) in Figure 3. This completes the proof of statement $(b)$ of Theorem 2.

4.3. Proof of statement $(c)$ of Theorem 2. The quadratic system (7) when $a \in$ $[(17 \sqrt{17}-71) / 32,0]$ has the following four equilibria

$$
\begin{aligned}
& p_{0}=(0,0), \quad p_{1}=\left(-\frac{1}{2+a},-\frac{1}{2+a}\right), \\
& p_{ \pm}=\left(\frac{( \pm R-1-2 a)( \pm 3 R-7-6 a)}{24-36 a}, \frac{\mp 3 R+7+6 a}{6 a-4}\right),
\end{aligned}
$$

where $R=\sqrt{4 a^{2}+16 a+1}$.

For $a \in((17 \sqrt{17}-71) / 32,0)$ the invariant algebraic curve $(8)$ is formed by an oval $O$ surrounding the hyperbolic unstable focus $p_{-}$and two components homeomorphic to straight lines, one of these components contains the hyperbolic stable node $p_{1}$ and the other contains the hyperbolic unstable node $p_{0}$. The remaining equilibrium $p_{+}$is a hyperbolic saddle. So the oval $O$ is a stable limit cycle, see Figure 4(b).

When $a \searrow(17 \sqrt{17}-71) / 32$ the oval $O$ collapses to the equilibrium $p_{-}$. Hence at $a=(17 \sqrt{17}-71) / 32$ there is a Hopf bifurcation, see Figure 4(a). When $a \nearrow 0$ the limit cycle $O$ ends in a heteroclinic loop with an orbit at infinity, see Figure $4(c)$.

In the local chart $U_{1}$ system (7) becomes

$$
\begin{aligned}
& \dot{u}=-2 u+v-(2+a) u^{2}-3 u v-a u^{3}, \\
& \dot{v}=-v\left(6+4(1+a) u+5 v+a u^{2}\right),
\end{aligned}
$$

and in the local chart $U_{2}$ is

$$
\begin{aligned}
& \dot{u}=a+(2+a) u+2 u^{2}+3 u v-u^{2} v, \\
& \dot{v}=-v\left(a+4(1+a) u+6 u^{2}+5 u v\right) .
\end{aligned}
$$

If $a \in[(17 \sqrt{17}-71) / 32,0)$ there are three infinite equilibria in $U_{1}$, namely $(-1,0)$ a hyperbolic saddle, $(0,0)$ a hyperbolic stable node, and $(-2 / a, 0)$ a hyperbolic saddle. If $a=0$ only the two first infinite equilibria remain in $U_{1}$. The origin of $U_{2}$ is a hyperbolic saddle only when $a=0$. 
In summary, with all the previous information we get the phase portraits in the Poincaré disc of system (7) in Figure 4. Hence statement $(c)$ of Theorem 2 is proved.

4.4. Proof of statement $(d)$ of Theorem 2. The quadratic system $(9)$ when $a \in[0,1 / 4]$ has the following four equilibria

$$
\begin{aligned}
& p_{ \pm}=\left(\frac{2 \pm \sqrt{4-7 a}}{7 a}, \frac{ \pm 3 \sqrt{4-7 a}-8}{14}\right), \\
& q_{ \pm}=\left(\frac{ \pm \sqrt{16-a}-4}{a}, \pm \frac{\sqrt{16-a}}{2}-1\right) .
\end{aligned}
$$

For $a \in(0,1 / 4)$ the invariant algebraic curve (10) is formed by an oval $O$ surrounding the hyperbolic stable focus $p_{+}$and two components homeomorphic to straight lines, one of these components contains the hyperbolic unstable node $q_{-}$and the hyperbolic saddle $q_{+}$. The remaining equilibrium $p_{-}$is a hyperbolic saddle. So the oval $O$ is an unstable limit cycle, see Figure 5(b).

In the local chart $U_{1}$ system (9) becomes

$$
\begin{aligned}
& \dot{u}=2 a u-14 a v-20 u^{2}-4 u v+(8-3 a) v^{2}-2 u v^{2}, \\
& \dot{v}=2 v\left(2 a-6 u-2 v-v^{2}\right),
\end{aligned}
$$

and in the local chart $U_{2}$ is

$$
\begin{aligned}
& \dot{u}=20 u-2 a u^{2}+4 u v+2 v^{2}+14 a u^{2} v+(-8+3 a) u v^{2} \\
& \dot{v}=2 v\left(-6 u+2 a u^{2}-2 u v-v^{2}\right) .
\end{aligned}
$$

If $a \in(0,1 / 4]$ there are two infinite equilibria in $U_{1}$, namely $(0,0)$ a hyperbolic unstable node and $(a / 10,0)$ a hyperbolic saddle. If $a=0$ only the $(0,0)$ is an infinite equilibrium in $U_{1}$ which, using blow ups (see for a precise definition [2]), is formed by two hyperbolic sectors separated by two parabolic sectors. These parabolic sectors contain a piece of the straight line of the infinity, see Figure 5(a). The origin of $U_{2}$ is a hyperbolic unstable node.

When $a \nearrow 1 / 4$ the oval $O$ collapses to the equilibrium $p_{+}$. Hence at $a=1 / 4$ there is a Hopf bifurcation, see Figure 5(c). When $a \searrow 0$ the equilibrium $p_{+}$goes to the infinite equilibrium $(0,0)$ of the chart $U_{1}$, and the limit cycle on the oval $O$ tends to an orbit of the elliptic sector of that infinite equilibrium; the equilibrium point $q_{\text {- tends to the }}$ equilibrium $(0,0)$ of $V_{1}$, see again Figure $5(a)$.

In short, with all the previous information we obtain the phase portraits in the Poincaré disc of system (9) in Figure 5 and, statement $(d)$ of Theorem 2 is proved. 


\section{Proof of Theorem 3}

5.1. Proof of statement $(a)$ of Theorem 3. The quadratic system (11) when $a \in$ $[3 \sqrt{7} / 2,4]$ has the following four equilibria

$$
\begin{aligned}
& p_{0}=(0,0), \quad p_{1}=\left(\frac{2}{a\left(a^{2}-16\right)},-\frac{1}{a}\right), \\
& p_{ \pm}=\left(-\frac{2 S}{\left(a^{2}-10 a+24\right)\left(a^{2}+16 a+48\right)^{2}}, \frac{S\left(26 a^{3}-22 a^{2}-360 a \pm R+576\right)}{3(a-6)(a-4)(a+4)^{2}(a+12)^{2}(13 a-24)}\right),
\end{aligned}
$$

where $R=\sqrt{\left(7 a^{2}-108\right)\left(13 a^{2}+28 a-96\right)^{2}}$ and $S=-35 a^{3}-86 a^{2}+504 a \pm R+1152$.

The proof of the existence of the algebraic limit cycle for system (11) under the conditions stated in statement $(a)$ of Theorem 3 was given in [19].

For $a \in(3 \sqrt{7} / 2,4)$ the invariant algebraic curve (12) is formed by an oval $O$ surrounding the hyperbolic unstable focus $p_{1}$ and one component homeomorphic to straight line which contain the hyperbolic nodes $p_{ \pm}, p_{-}$is unstable and $p_{+}$is stable. The remaining equilibrium $p_{0}$ is a hyperbolic saddle. So the oval $O$ is a stable limit cycle, see Figure $6(\mathrm{~b})$.

In the local chart $U_{1}$ system (11) becomes

$$
\begin{aligned}
& \dot{u}=\frac{(a-4)(a+4)^{2}(a+12) u-2(a-4)(a+4)^{2} v-8 a(a+4) u^{2}-20(a+4) u v+12 u^{3}}{a+4}, \\
& \dot{v}=\frac{2 v\left((a-4)(a+12)(a+4)^{2}-3(3 a-4)(a+4) u-14(a+4) v+6 u^{2}\right)}{a+4},
\end{aligned}
$$

and in the local chart $U_{2}$ is

$$
\begin{aligned}
& \dot{u}=-\frac{12-8 a(a+4) u+(a-4)(a+4)^{2}(a+12) u^{2}-20(a+4) u v-2(a-4)(a+4)^{2} u^{2} v}{a+4}, \\
& \dot{v}=\frac{2 v\left(6-3(a+4)(3 a-4) u+(a-4)(a+4)^{2}(a+12) u^{2}-14(a+4) u v\right)}{a+4} .
\end{aligned}
$$

If $a \in[3 \sqrt{7} / 2,2]$ there are three infinite equilibria in $U_{1}$, namely $\left(\left(a^{2}-16\right) / 2,0\right)$ a hyperbolic saddle, $(0,0)$ a hyperbolic stable node, and $\left(\left(48+16 a+a^{2}\right) / 6,0\right)$ a hyperbolic saddle. If $a=4$, using blow ups, the equilibrium point $(0,0)$ is formed by two hyperbolic sectors separated by two parabolic sectors and a piece of the straight line of the infinite is contained in both parabolic sectors, and the equilibrium $\left(\left(48+16 a+a^{2}\right) / 6,0\right)$ is a hyperbolic saddle, see Figure 6(c). The origin of $U_{2}$ is not an infinite equilibrium.

When $a \searrow 3 \sqrt{7} / 2$ the oval $O$ collapses to the equilibrium $p_{1}$. Hence at $a=3 \sqrt{7} / 2$ there is a Hopf bifurcation, see Figure 6(a). When $a \nearrow 4$ the equilibrium $p_{1}$ tends to the infinite equilibrium $(0,0)$ of the chart $U_{1}$, and the limit cycle $O$ tends to an orbit of 
the elliptic sector of that infinite equilibrium; the equilibrium $p_{+}$tends to the equilibrium $(0,0)$ of $V_{1}$, see again Figure $6(c)$.

In short, with all the previous information we obtain the phase portraits in the Poincaré disc of system (11) in Figure 6, and statement $(a)$ of Theorem 3 is proved.

5.2. Proof of statement $(b)$ of Theorem 3. The quadratic system (13) when $a \in$ $[3 / 2,2]$ has the following four equilibria

$$
\begin{aligned}
& p_{0}=(0,0), \quad p_{1}=\left(\frac{a+12}{R},-\frac{32 a(a+12)(2 a+3)}{R}\right), \\
& p_{ \pm}=\left(\frac{T \pm S}{U},-\frac{4 a(T \pm S)^{2}}{(a+12) U}\right)
\end{aligned}
$$

where $R=\sqrt{7\left(108+a^{2}\right)}, S=\sqrt{7(a+12)^{3}\left(259 a^{3}-336 a^{2}-36 a R^{2}+28476 a+75600\right)}$, $T=30240+1008 a+1302 a^{2}+119 a^{3}+504 a R+42 a^{2} R$ and $U=(7 a+3 R-42)\left(21 a^{2}+\right.$ $8 a R+42 a+12 R+1008)$.

The proof of the existence of the algebraic limit cycle of system (13) under the conditions of the statement $(b)$ of Theorem 3 was given in [1].

For $a \in(3 / 2,2)$ the invariant algebraic curve (14) is formed by an oval $O$ surrounding the hyperbolic stable focus $p_{+}$, and two component homeomorphic to straight lines, one of them contains the equilibria $p_{-}$and $p_{1}$, where $p_{-}$is a hyperbolic unstable node and $p_{1}$ is a hyperbolic saddle. The equilibrium $p_{0}$ is a hyperbolic saddle. Therefore the oval $O$ is an unstable limit cycle, see Figure 7(b).

In the local chart $U_{1}$ system (13) becomes

$$
\begin{aligned}
\dot{u}= & -\frac{1}{2(12+a)}\left(128 a^{2}\left(28728+22176 a+2730 a^{2}+476 a^{3}-108 R+864 a R+183 a^{2} R\right)+\right. \\
& 56 a\left(612+36 a+25 a^{2}+18 R+9 a R\right) u+2688(a-30) a^{3}(12+a) v+(7 a-42+3 R) u^{2} \\
& \left.+96 a(12+a)(-3+5 a) u v+6(12+a) u^{2} v\right), \\
\dot{v}= & -\frac{v}{2(12+a)}\left(-16 a\left(-252-126 a+28 a^{2}-18 R+9 a R\right)-(-42+7 a+3 R) u+\right. \\
& \left.336 a^{2}(12+a) v+6(12+a) u v\right),
\end{aligned}
$$


and in the local chart $U_{2}$ is

$$
\begin{aligned}
\dot{u}= & \frac{1}{2(12+a)}\left((-42+7 a+3 R) u+6(12+a) v+56 a\left(612+36 a+25 a^{2}+18 R+9 a R\right) u^{2}\right. \\
& +96 a(12+a)(-3+5 a) u v+128 a^{2}\left(28728+22176 a+2730 a^{2}+476 a^{3}-108 R+\right. \\
& \left.\left.864 a R+183 a^{2} R\right) u^{3}+2688(-30+a) a^{3}(12+a) u^{2} v\right), \\
\dot{v}= & \frac{v}{2(12+a)}\left((-42+7 a+3 R) u-6(12+a) v+16 a\left(-252-126 a+28 a^{2}-18 R+9 a R\right) u^{2}\right. \\
& \left.-336 a^{2}(12+a) u v\right) .
\end{aligned}
$$

If $a \in[3 / 2,2]$ there are two infinite equilibrium points in $U_{1}$, namely

$$
q_{ \pm}=\left(\frac{ \pm 12 V-28 a\left(25 a^{2}+9 a(R+4)+18(R+34)\right)}{7 a+3 R-42}, 0\right),
$$

where

$$
V=\sqrt{a^{2}\left((2052-a(47 a+540)) R^{2}+42(a+26)(a(a+36)+36) R+441(a(a+12)+84)^{2}\right)} .
$$

The equilibrium $q_{-}$is a hyperbolic saddle and $q_{+}$is a hyperbolic stable node. The origin of $U_{2}$ is a hyperbolic unstable node.

When $a \searrow 3 / 2$ the oval $O$ collapses to the equilibrium $p_{+}$. Hence at $a=3 / 2$ there is a Hopf bifurcation, see Figure $7(a)$. When $a \nearrow 2$ the limit cycle tends to a heteroclinic loop, see again Figure $7(c)$.

In short, with all the previous information we obtain the phase portraits in the Poincaré disc of system (13) in Figure 7. Therefore statement (b) of Theorem 3 is proved.

\section{Proof of Theorem 4}

The quadratic system (15) when $a \in[3 / 2,2]$ has the following four equilibria

$$
\begin{aligned}
& p_{0}=(0,0), \quad p_{1}=\left(\frac{5}{a}-\frac{1}{6}, 0\right), \\
& p_{ \pm}=\left(\frac{6-3 a \mp R}{2 a+24}, \frac{4 a\left(21 a^{2}+a(42 \pm 8 R)+12(84 \pm R)\right)}{a+12}\right),
\end{aligned}
$$

where $R=\sqrt{7\left(a^{2}+108\right)}$.

The proof of the existence of the algebraic limit cycle for system (15) under the condition stated in Theorem 4 was given in [19].

For $a \in(3 / 2,2)$ the invariant algebraic curve (16) is formed by an oval $O$ surrounding the hyperbolic stable focus $p_{1}$, and one component homeomorphic to a straight line which contains the equilibria $p_{ \pm}$, where $p_{+}$is a hyperbolic unstable node and $p_{-}$is a hyperbolic 
saddle. The equilibrium $p_{0}$ is a hyperbolic stable node. Therefore the oval $O$ is an unstable limit cycle, see Figure 8(b).

In the local chart $U_{1}$ system (11) becomes

$$
\begin{aligned}
& \dot{u}=-1344(a+30) a^{3}+48(5 a-3) a u+224(a-30)^{2} a^{2} v+3 u^{2}+52(a-30) a u v-u^{2} v, \\
& \dot{v}=-v\left(168 a^{2}+3 u+28(a-30) a v+u v\right),
\end{aligned}
$$

and in the local chart $U_{2}$ is

$$
\begin{aligned}
\dot{u}= & -3 u+v-48 a(-3+5 a) u^{2}-52(-30+a) a u v-1344(-30+a) a^{3} u^{3}- \\
& 224(-30+a)^{2} a^{2} u^{2} v \\
\dot{v}= & -v\left(3 u+v+168 a^{2} u^{2}+28(-30+a) a u v\right) .
\end{aligned}
$$

If $a \in[3 / 2,2]$ there are two infinite equilibrium points in $U_{1}$, namely

$$
q_{ \pm}=\left(8\left(-5 a^{2} \pm 3 \sqrt{a^{2}\left(2 a^{2}+20 a+1\right)}+3 a\right), 0\right) .
$$

Both equilibria are hyperbolic sadddles. The origin of $U_{2}$ is a hyperbolic stable node, see Figure 8.

When $a \nearrow 3 / 2$ the oval $O$ collapses to the equilibrium point $p_{1}$. Hence at $a=3 / 2$ there is a Hopf bifurcation, see Figure $8(a)$. When $a \nearrow 2$ the limit cycle on the oval $O$ tends to a heteroclinic cycle having an orbit at infinity, see Figure $8(c)$.

In summary, with all the previous information we obtain the phase portraits in the Poincaré disc of system (4) in Figure 8, and Theorem 4 is proved.

\section{ACKNOWLEDGEMENTS}

The first author is partially supported by the Ministerio de Economía, Industria y Competitividad, Agencia Estatal de Investigación grant MTM2016-77278-P (FEDER), the Agència de Gestió d'Ajuts Universitaris i de Recerca grant 2017SGR1617, and the H2020 European Research Council grant MSCA-RISE-2017-777911. The second author is partially supported by a FAPESP grant number 2017/20854-5. The third author is supported by NNSF of China No. 11971495.

\section{REFERENCES}

[1] M. Alberich-Carramiñana, A. Ferragut and J.Llibre, Quadratic planar differential systems having algebraic limit cycles, preprint 2019.

[2] M.J. Alvarez, A. Ferragut and X.Jarque, A survey on the blow up technique, Internat. J. Bifur. Chaos Appl. Sci. Engrg. 21 (2011), 3103-3118.

[3] J. Chavarriga, H. Giacomini and J. Llibre, Uniqueness of algebraic limit cycles for quadratic systems, J. Math. Anal. Appl. 261 (2001), 85-99.

[4] J. Chavarriga, J. Llibre And J. Sorolla, Algebraic limit cycles of quadratic systems, J. Differential Equations, 200 (2003), 206-244. 
[5] J. Chavarriga, J. Llibre and J. Moulin Ollagnier, On a result of Darboux, LMS J. of Computation and Mathematics 4 (2001), 197-210.

[6] C. Christopher, J. Llibre And G. Świrszcz, Invariant algebraic curves of large degree for quadratic systems, J. of Math. Anal.Appl., 303 (2005), 450-461.

[7] F. Dumortier, J. Llibre And J. C. Artés, Qualitative theory of planar differential systems, Universitext, Springer, New York, 2006.

[8] F. Dumortier, R. Roussarie and C. Rousseau, Hilbert's 16th problem for quadratic vector fields, J. Differential Equations 110 (1994), 86-133.

[9] R.M. Evdokimenco, Construction of algebraic paths and the qualitative investigation in the large of the properties of integral curves of a system of differential equations, Differential Equations 6 (1970), 1349-1358.

[10] R.M. Evdokimenco, Behavior of integral curves of a dynamic system, Differential Equations 9 (1974), 1095-1103.

[11] R.M. Evdokimenco, Investigation in the large of a dynamic system, Differential Equations 15 (1979), 215-221.

[12] V.F. Filiptsov, Algebraic limit cycles, Differential Equations 9 (1973), 983-986.

[13] B.R. de Freitas, J. Llibre And J.C. Medrado, Limit cycles of continuous and discontinuous piecewise-linear differential systems in $\mathbb{R}^{3}$, J. Comput. Appl. Math. 338 (2018), 311-323.

[14] Yu Ilyashenko, Centennial history of Hilbert's 16-th problem, Bull. Amer. Math. Soc. 39 (2002), 301-354.

[15] J. LI, Hilbert's 16th problem and bifurcations of planar polynomial vector fields, Internat. J. Bifur. Chaos Appl. Sci. Engrg. 13 (2003), 47-106.

[16] Y.A. Kuznetsov, Elements of applied bifurcation theory, Third edition. Applied Mathematical Sciences 112. Springer-Verlag, New York, 2004.

[17] J. LliBRe AND J. ITIKAWA, Limit cycles for continuous and discontinuous perturbations of uniform isochronous cubic centers, J. Comput. Appl. Math. 277 (2015), 171-191.

[18] J. Llibre And D. Schlomiuk, The geometry of differential quadratic systems with a weak focus of third order, Canadian J. Math. 56 (2004), 310-343.

[19] J. Llibre AND G. Świrszcz, Classification of quadratic systems admitting the existence of an algebraic limit cycle. Bull. Sci. Math. 131 (2007), 405-421.

[20] L. Markus, Global structure of ordinary differential equations in the plane: Trans. Amer. Math Soc. 76 (1954), 127-148.

[21] D. A. Neumann, Classification of continuous flows on 2-manifolds, Proc. Amer. Math. Soc. 48 (1975), 73-81.

[22] M.M. Peiхото, On the classification of flows on 2-manifolds. Academic, New York, pages 389-419, 1973. Dynamical systems (Proc. Sympos., Univ. Bahia, Salvador, 1971).

[23] QIN YUAN-Xun, On the algebraic limit cycles of second degree of the differential equation $d y / d x=$ $\sum_{0 \leq i+j \leq 2} a_{i j} x^{i} y^{j} / \sum_{0 \leq i+j \leq 2} b_{i j} x^{i} y^{j}$, Acta Math. Sinica 8 (1958), 23-35.

[24] A.I. YABLONSKII, Limit cycles of a certain differential equations, Differential Equations 2 (1966), 335-344 (In Russian). 
Departament de Matemàtiques, Universitat Autònoma de Barcelona, 08193 Bellaterra, Barcelona, Catalonia, Spain

Email address: jllibre@mat.uab.cat

Departamento de Matemática, Instituto de Ciências Matemáticas e Computação, Universidade de São Paulo, Avenida Trabalhador São Carlense, 400, 13566-590, São Carlos, SP, BRAZIL.

Email address: regilene@icmc.usp.br

School of Mathematics (Zhuhai), Sun Yat-sen University, Zhuhai 519082, Guangdong, People's Republic of China

Email address: mcszyl@mail.sysu.edu.cn 